\title{
Research on the Characteristics of the Rural Landscape Based on the System of Landscape Evaluation
}

\author{
Gui-ju Lv ${ }^{1}$, Da-liang Liu ${ }^{3}$, Ming-yuan Zhao ${ }^{2}$, Lan-yong Zhao ${ }^{2,}$ * \\ ${ }^{1}$ College of Horticultural Science and Engineering, Shandong Agricultural University, Taian, China \\ ${ }^{2}$ College of Forestry, Shandong Agricultural University, Taian, China \\ ${ }^{3}$ College of Art, Shandong Jianzhu University, Jinan, China
}

\section{Email address:}

sdzly369@163.com (Lan-yong Zhao)

${ }^{*}$ Corresponding author

\section{To cite this article:}

Gui-ju Lv, Da-liang Liu, Ming-yuan Zhao, Lan-yong Zhao. Research on the Characteristics of the Rural Landscape Based on the System of Landscape Evaluation. International Journal of Science, Technology and Society. Vol. 5, No. 6, 2017, pp. 186-192. doi: $10.11648 /$ j.jijsts.20170506.13

Received: September 15, 2017; Accepted: October 1, 2017; Published: October 27, 2017

\begin{abstract}
Rural landscape is a forward field in the scientific research on scenery. Under the circumstance of rapid urbanization in China, the implementation of rural landscapes is extremely urgent. The characteristics of country landscapes have been the accelerator of the sound and sustainable development of the rural economy and society. As a result, the research object of study is the five natural villages in Xiying county in the central part of Shandong province. Based on the classification of the rural landscapes, and combined with expert opinions, the comprehensive evaluation system for rural landscapes has been built, which consists of total target layer, factor index layer and function index layer. Besides, the study analyses the characteristics of the five rural landscapes in the investigation area based on the system. By defining the characteristics of the landscapes, the vitality and attraction have been inspired. Meanwhile, the study provides reference for the similar study.
\end{abstract}

Keywords: Rural Landscapes, Index System, Landscape Evaluation, Characteristics

\section{Introduction}

Village is the remote origin and base of our rural life, which carries on the long agricultural civilization. It is also a place where the essence and temperament of the local people could be found. The traits of village are not only the absolute force to balance the phenomenon that the current architectural culture, urban space and structural shape are almost the same, but the powerful core of rural ecology and the development of economy and culture.

President Xi Jinping paid high attention to the reflection and inheritance of regional culture, stressing that the strong and prosperity of a country or a nation relies on the flourishing of its culture. The realization of China Dream would never come true without inheritance and development of civilization as well as promotion and prosperity of culture. In addition, Li Keqiang added that the development of urbanization should not make all the towns the same. On the contrary, it has to inherit its culture and build up its unique characteristics.
Otherwise, it cannot make use of its own advantages and constructs all cities in a same way. Mr. Nan Huaijin has said that the aim of reading thousands of years' history is nothing more than to become rich and famous; The truth of philosophy that has been pursued for too many years can be nothing but the life of poetry, wine and countryside. Countryside is an idyllic dream of Chinese people, which is thought to possess the order of family ethics, the thriving of folk craft, the poetic scene of bridges and flowing water, the amiable rural scenery, the traditional rural culture, the idyllic way of lifestyle. All of these constitute the idyllic dream of Chinese people.

All countries are unified in the common characteristics of the region, and at the same time, carrying unique local features. Common traits are the study of the characteristics of rural landscape in the study area, while personality traits are the study of landscape features of each independent village on the premise of common traits. The paper will study the unique traits 
of rural landscapes through the methods of the comprehensive evaluation system for rural landscapes, find out the differences among villages, and define the characteristics of rural scenery. By doing so, the study hopes to bring the charm and vitality to the villages, and leads to the development of rural areas.

\section{Overview of the Research Area}

The provincial capital city Jinan is one of the most important economic centers in China, as well as a national historical and cultural city with a title of the first group of top tourist cities. It is also one of the birthplace of a prehistoric culture--Longshan Culture and a prosperous metropolis in the middle and lower reaches of Yellow River.

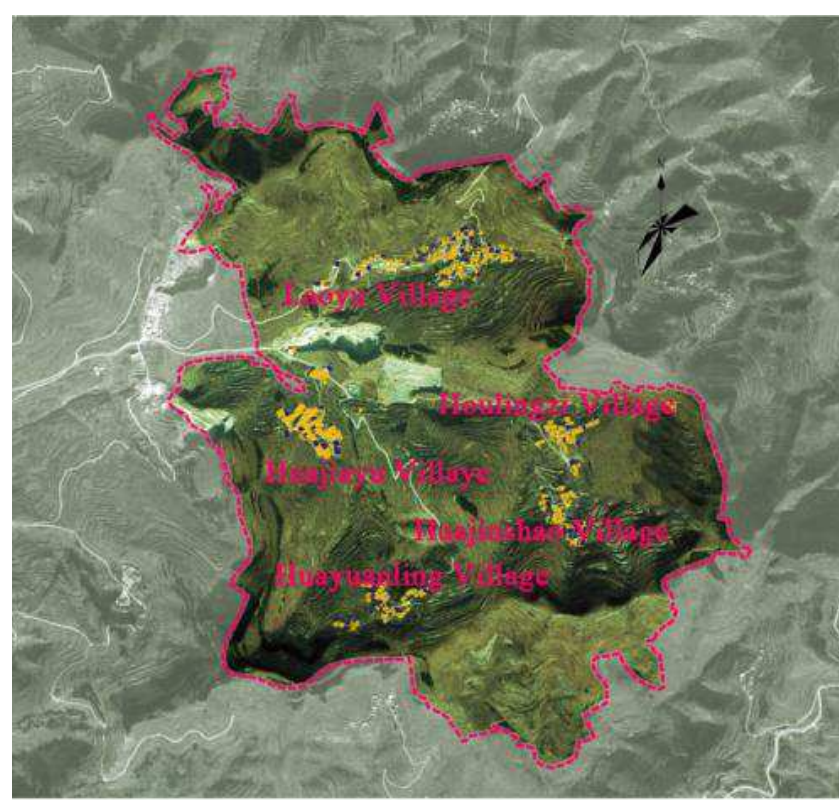

Figure 1. Research Field Location Map.

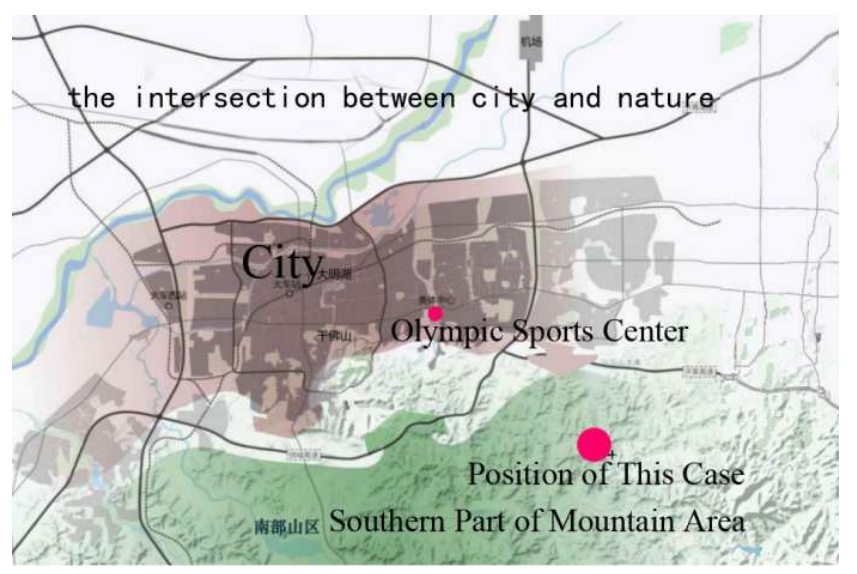

Figure 2. Research Villages Location Map.

Besides, Jinan is north of Shanghai and Ningbo, south of Beijing and Tianjin, east and west connecting the Shandong Peninsula and Central China Region, it is an important traffic hinge in circum-Bohai area and the line of Beijing and Shanghai. Jinan is located in the connecting zone between the low-hilly land in the middle and southern Shandong Province and the alluvial plain in the southwestern of Shandong Province with a terrain high in south and low in north. The research of this paper includes some typical villages of hilly and mountain area in southern Jinan ( Figure 1 and Figure 2), which locate in the intersection between city and nature. With a unique geographic location, they are situated in Mount Tai extension and surrounded by ravines, gullies and numerous mountains. Villages are the backyard of a city, because of its beautiful scenery and natural environment. The research field includes Laoyu Village, Huajia Valley, Huayuanling, Huajinxiao and Houlingzi, covering an area of $5000 \mathrm{mu}$ (about 3333333.33 squire meter).

\section{Data Source and Research Method}

\subsection{Data Source}

The research method is determined by prof. Chen Zhihua who majors in architecture in Tsinghua university. He advocates the case study of the village, and supports the use of a empirical fieldwork method with strong authenticity to obtain valuable first-hand information. Therefore, the data of this paper is mainly from the on-site research and interview questionnaires of the professional teachers and students. The research carried out in 2016 from October to November as well as a research from April to May in 2017. First of all, all of the indicators' weight of the survey can be determined through the use of AHP method [1]-[3], so that each factor has a scientific quantitative. Then, according to the characteristics of the rural landscape in the middle of mountains in Shandong province, reasonable and scientific scoring criteria has been made in accordance with each indicator. Finally, according to the scoring standard, the comprehensive method is used to assign the index of the typical rural landscape index in the middle of Shandong province. The general index value and the index of the rural landscape evaluation are calculated by the formula, and the factor index diagram has been drew to compare and analyze in an direct way [4]-[6].

\subsection{Research Method}

\subsubsection{The Construction of the Evaluation System for the Rural Landscapes}

Taking the five natural villages in Xiying county as the research object,the study is based on the classification of the rural landscapes. It divides the rural landscapes into five parts, namely, economic production, residential life, natural habitat, spiritual culture and pattern formation. After combining with expert opinions, the nine factor index layers come into being, including village pattern, architectural features, courtyard space, streets and alleys space, cultural assembly space, plant landscapes, mountain landscapes, water landscapes and cultural characteristics.

Comprehensive evaluation system for rural landscapes has been built, which consists of total target layer, factor index layer and function index layer. The choice of function index layer focuses on the options that facilitate people to judge intuitively, for example, the ecology, beauty and long history of the landscape. The function layer is determined. The functional indicators are determined according to different 
factors, and in this evaluation system that has been talked about, there are totally16 functional index layers including landscape features, residential buildings and so forth. Then, the comprehensive evaluation system for rural landscapes has been built, consisting of total target layer, factor index layer and function index layer. (Table 1).

Table 1. Evaluation System of Rural Landscapes.

\begin{tabular}{lll}
\hline General Object & Factor Index Level & Function Index Level \\
\hline & Village Pattern & Security and Convenience \\
& Rural Landscape & Speciality \\
& Residential Architecture & $\begin{array}{l}\text { Durability, Aesthetics and } \\
\text { Speciality }\end{array}$ \\
& Streets and Lanes & Beautiful Form and Rich \\
General & Landscape & Space \\
Evaluation & Cultural Assembling & Space Infection \\
Of Rural & Space & Ecology and Appreciation \\
Landscape & Mountain Landscape & Ecology and Appreciation \\
& Water Landscape & Season and Coverage \\
& Plant Landscape & Cultural Attraction and \\
& Spiritual Culture & Cultural localization \\
\hline
\end{tabular}

\subsubsection{Evaluation and Assignment of Rural Landscape}

The evaluation system mainly adopts a standard that consists of 5 grades, namely very satisfied, satisfied, general, unsatisfied, and very unsatisfied. [7]. Then they would be scored respectively $5,4,3,2$, and 1 .

The security of the village is the most basic requirement for the formation and development of the countryside. [8]. Generally speaking, rural location is a long way from the main road for the consideration of security. At the same time, there are water, mountains and dense greenery around the village to protect it from the outside intrusion. However, excessive safety can also cause inconvenience to villagers, especially when today's information exchange and rural development rely heavily on the main roads that linking countryside and city. Only by striking a balance between the two can a village be the best.

The characteristics of agricultural landscapes include two aspects. On the one hand, it is the characteristics of crops growth conditions such as terrace with elevation differences, waterside and so on. On the other hand, it is the characteristics of the crop species such as the rarity of it. When both the location and species characteristics of the crop are strong, it gets the highest rating.

The long tradition of residential buildings is based on the age of the village houses in the middle of Shandong province, and the scores are determined in the order of history. The residential buildings built more than 100 years ago, 50 to 70 years ago, 50 to 70 years ago, 20 to 50 years ago and less than 20 years ago are evaluated respectively according to the grade of $5,4,3,2,1$. The beauty of residential buildings refers to the degree of beauty left by the sensory impression of the whole house, including style, color, layout, decoration. The characteristics of residential buildings refer to the layout, wall, roof, doors and windows, and decoration of the building.

The beauty of street landscapes is the form of material combination and the beauty of color and texture of the bottom interface and the vertical interface of the street. The richness of street space is the various space types formed by the proportion of width and height of street roads, including open space, semi-open space and closed space. Walls, mountains, and fields also form rich street space.

Cultural infection refers to the environment elements in the area making people grow site identity and think of the previous village activities. [9]. The village with rich historical factors is very impressive, which makes people have a rich association with the previous rural activities, and therefore has high infection.

The evaluation of the mountain landscape, water landscape and plant landscape contains the integrity of the mountain and the cover of the plant (Table 2).

Table 2. Function Index Meaning of Mountain, Water and Plant Landscape.

\begin{tabular}{ll}
\hline & Ecology \\
\hline Mountain Landscape & Mountain Integrity and Plant Coverage \\
Water Landscape & Clean, pollution-free and thriftiness \\
Plant Landscape & Greening and Plant Coverage \\
\hline
\end{tabular}

Ornamental Value
Richness of mountainous terrace and mountainous plants
Richness of water revetment line and water landscape
Ornamental shape of plants in four seasons

Plant coverage refers to the percentage of the projected area of greening in the rural settlement and its around area. According to the Regulations on Inspection and Acceptance of National Villages with Beautiful Environmental (Trial), the mountain forest coverage rate is regulated as $70 \%, 40 \%$ of the hills, plain $10 \%$. Hence, considering the actual situation of plant coverage in rural areas, the scope of the survey within the rural green coverage rate is divided as follows: green coverage rate above $80 \%$ is 5 points of the evaluation, green coverage between $70 \%-80 \%$ is 4 points of the evaluation, green coverage between $60 \%-70 \%$ is 3 points of the evaluation, green coverage between $50 \%-60 \%$ is 2 points of the evaluation, green coverage under $50 \%$ is 1 point of the evaluation level.

Spiritual culture of villages includes the attraction of culture and cultural localization. The former refers to the distinct cultural characteristic, strong life atmosphere and the villagers' simple enthusiasm. Cultural localization is the most special part of a culture.

\subsubsection{Calculation Method of Rural Landscape Evaluation}

By means of AHP method (Analytic Hierarchy Process), each index of factor index layer and function index layer is quantitatively quantified and its index weight is determined (Table 3). 
Table 3. Weight of Comprehensive Assessment Indexes of Rural Landscape in the middle of Shandong Province Mountain Area.

\begin{tabular}{|c|c|c|}
\hline Level of the General Object & Factor Index Level and its Weight & Functional Index Level and its Weight \\
\hline \multirow{16}{*}{$\begin{array}{l}\text { A } \\
\text { General Evaluation of } \\
\text { Rural Landscape }\end{array}$} & B1 Village Pattern 0.0747 & C1 Security and Convenience in Traffic 1.0000 \\
\hline & B2 Rural Landscape 0.0823 & C2 Speciality 1.0000 \\
\hline & & C3 Durability 0.2958 \\
\hline & B3 Residential Building 0.2036 & C4 Aesthetics 0.3934 \\
\hline & & C5 Characteristics O.3108 \\
\hline & \multirow{2}{*}{ B4 Landscape of Streets and Lanes 0.1081} & C6 Ornamental Shape 0.4722 \\
\hline & & C7 Space Richness O.5278 \\
\hline & B5 Cultural Assembling Space 0.0827 & C8 Space Infection 1.0000 \\
\hline & \multirow{2}{*}{ B6 Mountain Landscape 0.1536} & C9 Mountain Ecology 0.6273 \\
\hline & & C10 Mountain Landscape 0.3727 \\
\hline & \multirow{2}{*}{ B7 Water Landscape 0.1007} & C11 Water System Ecology 0.6526 \\
\hline & & C12 Water System Landscape 0.3474 \\
\hline & \multirow{2}{*}{ B8 Plant Landscape 0.1120} & C13 Plant Season 0.4017 \\
\hline & & C14 Plant Coverage 0.5983 \\
\hline & \multirow{2}{*}{ B9 Spiritual Culture 0.0823} & C15 Cultural Attraction 0.4282 \\
\hline & & C16 Cultural Lacation 0.5718 \\
\hline
\end{tabular}

Referring to the comprehensive evaluation formula of Yang zhijie's investigation and analysis and evaluation of rural settlements in Shanghai [11]-[12], we construct a comprehensive evaluation formula of rural landscape in luzhong district, namely:

$$
\mathrm{P}=\sum_{j=1}^{m}\left(\sum_{i=1}^{n} \mathrm{C}_{\mathrm{i}} \mathrm{M}_{\mathrm{i}}\right) \mathrm{R}_{\mathrm{j}}
$$

$P$ is the total score; $C_{i}$ is the score of functional index; $M_{i}$ is the weight of function index; $R_{j}$ is the weight of the factor index; I is the number of functional indicators; $\mathrm{J}$ is the number of factor index.

In this index system, $\mathrm{i}$ takes 16 . and $\mathrm{J}$ is the number of factor indexes. In this index system, $\mathrm{j}$ takes 9 , and the evaluation total score is 5 . In combination with expert opinion and the actual situation of the rural areas in the mountainous area, the rural landscape is satisfactory when its score is more than 3 points.

On the basis of the evaluation and assignment of a series of tables, summarizing the scores that professional teachers and students have given, combined with expert scoring and opinion, we finally determine the rural settlement landscape function index score list (Table 4).

Table 4. Values of Rural Settlement Landscape in Rural Mountainous Landscape of Middle Shandong Province.

\begin{tabular}{|c|c|c|c|c|c|c|c|c|c|c|c|c|c|c|c|c|}
\hline & $\mathrm{C1}$ & $\mathrm{C} 2$ & $\mathbf{C 3}$ & $\mathrm{C4}$ & C5 & C6 & C7 & $\mathbf{C 8}$ & C9 & $\mathrm{C10}$ & C11 & $\mathrm{C12}$ & $\mathrm{C} 13$ & $\mathrm{C14}$ & $\mathrm{C} 15$ & $\mathrm{C16}$ \\
\hline Laoyu Village & 4 & 3 & 4 & 3 & 4 & 4 & 4 & 3 & 3 & 2 & 1 & 1 & 2 & 2 & 2 & 2 \\
\hline Huajiayu Village & 3 & 4 & 3 & 3 & 3 & 3 & 3 & 2 & 3 & 2 & 2 & 1 & 2 & 3 & 2 & 2 \\
\hline Huayuanling Village & 2 & 3 & 4 & 3 & 4 & 4 & 4 & 2 & 3 & 4 & 2 & 2 & 2 & 3 & 2 & 2 \\
\hline Houlingzi Village & 3 & 4 & 2 & 1 & 1 & 2 & 3 & 1 & 2 & 2 & 1 & 1 & 2 & 3 & 1 & 1 \\
\hline Huajinshao Village & 1 & 2 & 1 & 2 & 2 & 2 & 4 & 1 & 4 & 4 & 2 & 2 & 3 & 2 & 2 & 2 \\
\hline
\end{tabular}

Applying the comprehensive evaluation formula of rural landscape in Shandong district, we get the score of 9 factor indexes and the total score of comprehensive evaluation of the following five places, namely Lao Yu Village, Hua Jiayu, Hua Yuanling, Hou Lingzi and Hua Jinshao. (Table 4)

Taking the residential building of Lao Yu Village as an example, the calculation method of factor index score is explained as follows.

The score of residential building $=$ $(4 * 0.2958+3 * 0.3934+4 * 0.3108) * 0.2136=0.7343$

The comprehensive score of the landscape in Laoyu Village is the sum of values of the nine factor indicators of the village.
In other words, the comprehensive score of the landscape in Laoyu village $=$ score for pattern and convenience + score for agricultural landscapes+score for residential building+score for streets landscape+score for cultural gathering space ++ score for the mountain landscape + score for the water landscape+score for plant landscape+score for spiritual culture

$$
\begin{aligned}
& =0.2989+0.2469+0.7342+0.4324+0.2481 \\
& +0.4036+0.1007+0.2240+0.1646 \\
& =2.8534
\end{aligned}
$$


Table 5. Total Points of Rural Landscape Factor Index Score and Comprehensive Evaluation in the middle of Shandong Province Mountain Area.

\begin{tabular}{llllll}
\hline Factor Index & Laoyu Village & Huajiayu Village & Huayuanling Village & Houlingzi Village & Huajinshao Village \\
\hline B1 & Layout and Convenience & 0.2988 & 0.2241 & 0.1494 & 0.2241 \\
B2 & Rural Landscape & 0.2469 & 0.3293 & 0.2469 & 0.0747 \\
B3 & Residential Landscape & 0.7343 & 0.6108 & 0.7343 & 0.1646 \\
B4 & Streets and Lanes Landscape & 0.4324 & 0.3243 & 0.4324 & 0.2638 \\
B5 & Cultural Assembling Space & 0.2481 & 0.1654 & 0.1654 & 0.2733 \\
B6 & Mountain Landscape & 0.4036 & 0.4036 & 0.5180 & 0.0827 \\
B7 & Water Landscape & 0.1007 & 0.1664 & 0.2014 & 0.3072 \\
B8 & Plant Landscape & 0.2240 & 0.2911 & 0.2911 & 0.1007 \\
B9 $\quad$ Intangible Cultural Heritage & 0.1646 & 0.1646 & 0.1646 & 0.2911 \\
Composite Score & 2.8534 & 2.6796 & 2.9035 & 0.0823 \\
\hline
\end{tabular}

\section{Results and Analysis}

\subsection{Comprehensive Analysis}

The comprehensive evaluation of rural landscape in the mountainous areas in central Shandong is below 3 points,so the overall landscape of the countryside is not that satisfactory and there is much to be improved. The total score of Laoyu Village, Huajia Valley, Huayuanling is above 2.5, which shows that the overall landscape of these three villages is relatively satisfied. The value of the Huajinshao is 2.3089 , between 2 and 2.5, which is of medium level. The total value of Houlingzi is 1.954 5, which is less satisfactory. Make a comprehensive chart of the scores of nine factors of rural landscape (Figure 3). The five villages are then rated according to the five main meanings of the five villages. There is best (first), second-best (second), better (third), worst (last). (Table 6).

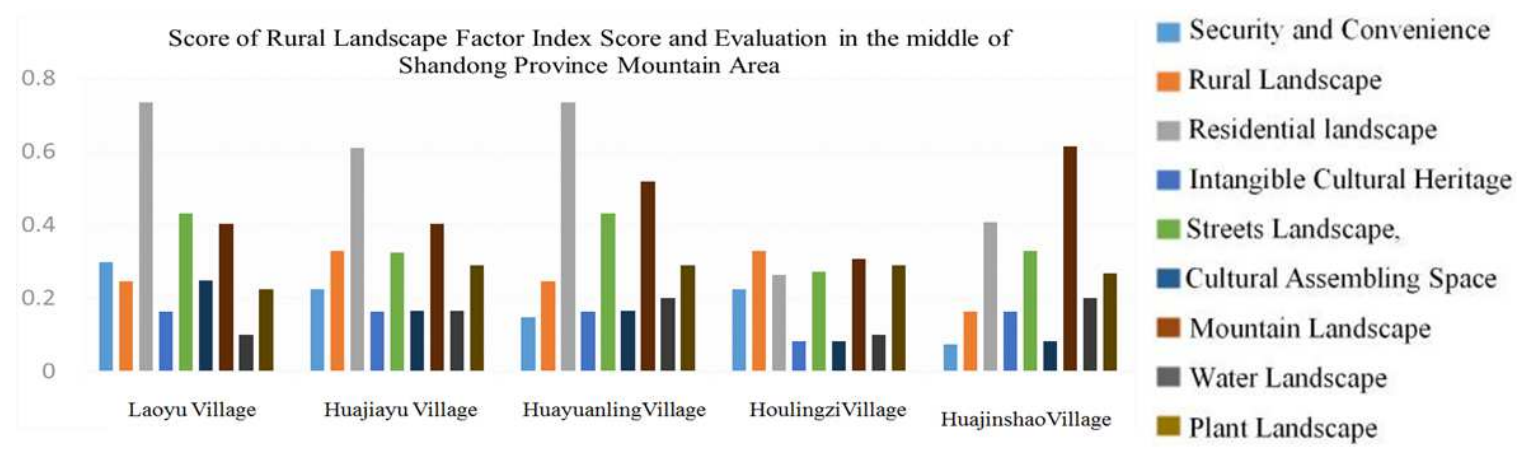

Figure 3. Score Map of Rural Landscape Factor Index Score and Evaluation in the middle of Shandong Province Mountain Area.

Table 6. Comparison of Meanings and Characteristics of Rural Landscape in the middle of Shandong Province Mountain Area.

\begin{tabular}{|c|c|c|c|c|c|}
\hline Factor Index & Laoyu Village & Huajiayu Village & Huayuanling Village & Houlingzi Village & Huajinshao Village \\
\hline Security and Convenience & $\sqrt{ }$ & $※$ & $*$ & (0) & \\
\hline Rural Landscape & * & $※$ & * & $\sqrt{ }$ & (0) \\
\hline Residential Life & $\sqrt{ }$ & $*$ & $※$ & (0) & \\
\hline Natural Environment & & * & $※$ & () & $\sqrt{ }$ \\
\hline Intangible Cultural Heritage & $※$ & $※$ & $※$ & (0) & $※$ \\
\hline
\end{tabular}

$\sqrt{ }$ Best $※$ Second-Best * Common $\bigcirc$ Worst

Judging from the above-mentioned information, the most striking features of the five places are the security and convenience of Laoyu Village, the agricultural landscapes of Houlingzi, and the natural landscapes of Huajinshao. There are no outstanding features of Huayu Valley and Huayuanling. The evaluation results of each factor of all five villages are not so bad. Among them, Huayuanling focuses more on living and natural environment, and Huajia Valley focuses more on agricultural landscape and security.

\subsection{Case Study}

In order to find the characteristics of each village,Security and convenience (A), rural landscape (B), residential landscape (C), streets landscape (D), cultural assembling space $(E)$, mountain landscape $(F)$, water landscape $(G)$, plant landscape $(\mathrm{H})$, intangible cultural heritage (I) the nine attributes of each village are taken as index scores

\subsubsection{Characteristics Analysis of Laoyu Village}

The safety and convenience of Laoyu Village (Figure 4) are the highest among the five villages. It is generally believed that it is appropriate to walk 20 to 30 minutes from the village to the main street of the outer city. If it is too close, the tranquility of the country is lost. If too far, it is inconvenient for the villagers and the outsiders. The main roads and main spaces of Laoyu Village are flat. At the same time, the distance between Laoyu Village and four other villages are about 1500 meters. The transportation of the village is comparatively 
convenient. Compared with other villages, Laoyu Village has the highest rate of traffic convenience, cultural meeting space and residential landscape, which is the most important feature of central village.

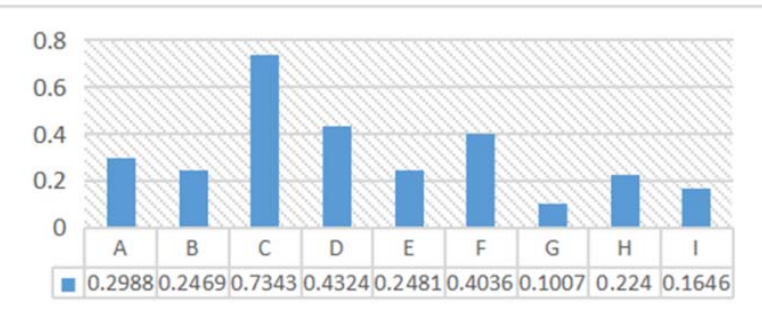

Figure 4. Scores Map of Landscape Factor Index of Laoyu Village.

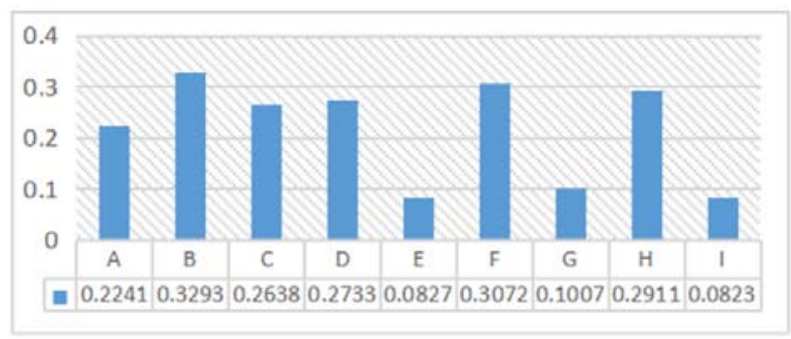

Figure 5. Scores Map of Landscape Factor Index of Houlingzi Village.

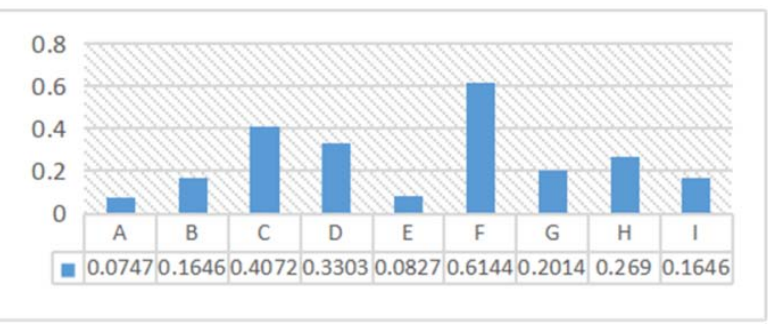

Figure 6. Scores Map of Landscape Factor Index of Huajinshao village.

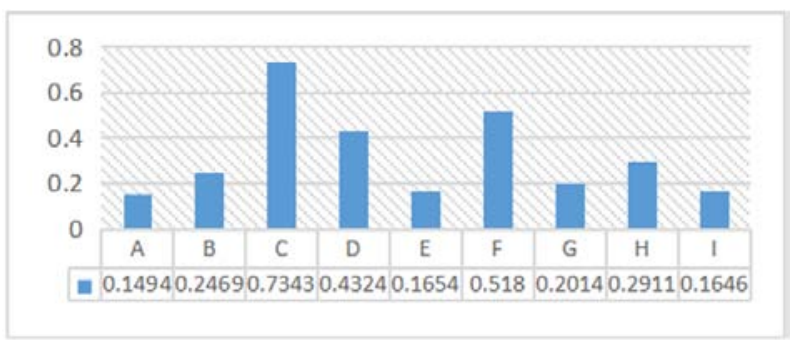

Figure 7. Scores Map of Landscape Factor Index of Huayuanling village.

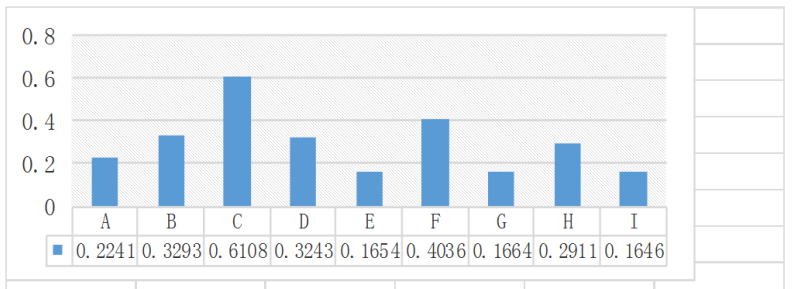

Figure 8. Scores Map of Landscape Factor Index of Huajiayu village.

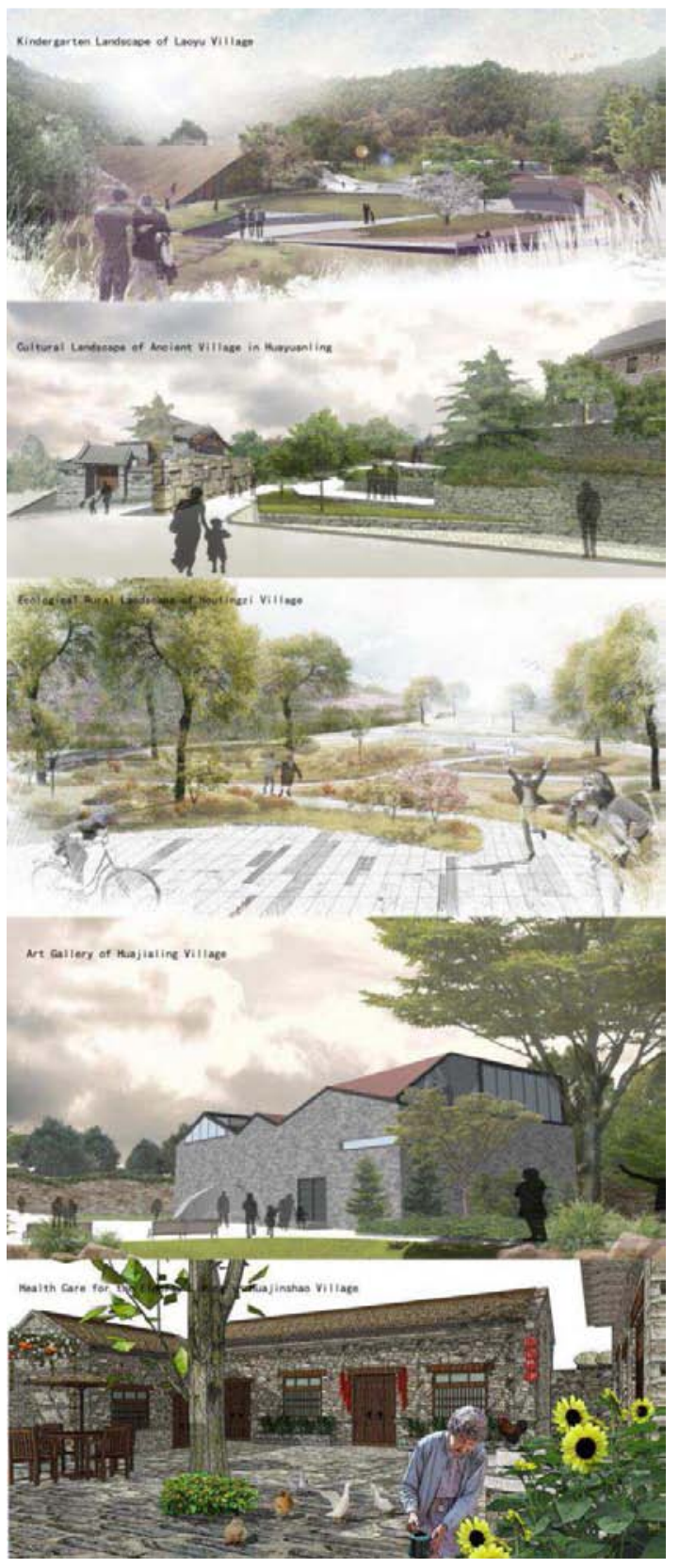

Figure 9. Landscape Characteristics of Five Natural Villages.

Therefore, if it is defined as the village with comprehensive functions, it has to satisfy the various functions of education, health care, children's amusement, and mountain sightseeing.

\subsubsection{Characteristics Analysis of Houlingzi Village}

The total score of landscape evaluation of Houlingzi (Figure 5) is the lowest. The scores of the other factors are the lowest except for the agricultural landscape. Agricultural landscape features Houlingzi. Although the kinds of crops are similar to those of other villages, the village has a large and concentrated terraced landscape, which is very unique in mountainous countrysides. However, the agricultural land in other villages is scattered. Therefore, the orientation of the landscape architecture of the village is to take the opportunity of developing modern organic agriculture, to optimize the rural natural habitat and residential landscape, and to improve 
the landscape quality of the countryside.

\subsubsection{Characteristics Analysis of Huajinshao}

Huajinshao, (Figure 6) has a distinct characteristic and has the best natural environment. It is a suitable place for health care with mountains surrounded by a wide view. And there is flourishing plants, cozy sunshine in the winter, cool breeze in the summer. During the construction of landscape, part of the families are either moved out or merged together in order to transform and upgrade the dwelling houses. The stone houses are rented in this area to urban residents, with emphasis on health care so that they can attract groups who expect ecological endowment.

\subsubsection{Characteristics Analysis of Huayuanling Village}

The evaluation of the nine factor indicators of Huayunaling (Figure 7) are not particularly outstanding, but are comparatively good, especially living and natural habitat, which is second only to Huajinshao where living stander is the best. Combined with other rural positioning, this village can be positioned as an ancient village cultural experience. Therefore, we need to organize the street space, repair and highlight architectural features, at the same time, create a sense of space, and increase the total amount and form of greening, in order to create a beautiful countryside with strong sense of history.

\subsubsection{Characteristics Analysis of Huajiayu Village}

The village pattern of Huajia Valley (Figure 8) is convex with a wide view and high elevation difference. And it has three rich hyperopia scenes. There are rich perception of different degrees, of different distances and different angles between people and mountains and villages. Nature is the best place and source of art. The growth of all things varies with the weather, time and positions, but it is harmonious and beautiful. People in such a natural environment are more likely to be inspired with a lot of creative ideas, purify the mind and have a physical and mental pleasure. In addition to relatively pretty living landscape and natural habitats, there is a large area of terraced landscape with prominent position and high evaluation. Compared to other rural areas, Huayan Valley is best suited to develop the art countryside. Artistic means can be used to enhance the charm of the countryside and the value of the industry, providing the functions related to the arts including art exhibition, art painting, art creation, artist design studio, art study and so on.

\section{Conclusion}

Since the 21 st century, the rural industrial structure, village type, land use, material space, and people's lifestyles are undergoing profound changes. Therefore, the village can not just follow the step of the city to lose their own characteristics, and can not stand still to avoid development. The development of the countryside must be the essence and the core of the creation to achieve the coordinated development of rural ecology and cultural economy, of which ecology and culture in the rural development are indispensable. It is the fertility to foster rural characteristics. With the adaptation between human and nature, the village landscape will be printed with local culture brand and obtain a mutual harmony, thus the rural landscape will show the nature of human culture.

The characteristics of the rural landscape are the root cause of the vitality, attraction and endurance of the countryside. Based on the individualized characteristics of the rural landscape, great importance has been attached to the integration of resources, transformation of the environment and realization of the activation and development of the countryside. Through the use of the comprehensive evaluation model of rural landscape, this paper studies the rural landscape in the mountain area, and captures the rich connotation of the value of rural landscape in general. Through the visual comparison between the various indexes, the landscape characteristics of each country (Figure 9) can be analyzed and found, as well as the advantages and disadvantages in the hope of finding effective ways and methods for the research and development of rural landscapes.

\section{References}

[1] Chen Ying, Huang Chengfeng. Application of AHP and Fuzzy Synthetically Evaluation in Humanities Landscape of Superhighway [J]. Environmental Protection Science. 2007, 33(3):17-25

[2] Xie Hualin, Liu Liming, Zhao Yingwei. Studies on the Indicator System of Rural Landscape Evaluation and Evaluating Method [J]. Research of Agricultural Modernization. 2003, 24(2):95-102

[3] Liu Zhihao, Jin Qiming. Study on the Types of Rural Cultural Landscape and Its Evolution [J]. Journal of Nanjing Normal University (Natural Science). 1999, 22(4):121-128

[4] Shen Mingrui, Zhang Jingxiang. China's Rural Transformation and Revival in the Context of New Urbanization [J]. City Planning Review. 2015(01):42-47

[5] Hu Ruiyi, Chen Dongtian, Yu Caiyun, Zhang Ying. Research on Design of Rural Landscape on Low-cost Concept [J]. Shandong Forestry Science and Technology. 2017(08)

[6] Han Yue, Tian Dafang. Construction of Evaluation Index System of Rural Tourism Landscape Attraction [J]. Shanxi Architecture. 2017(01)

[7] Shao Juehan, Liu Binyi. Analyzing the Visual Perception of Rural Landscape [J]. Chinese Landscape Agriculture. 2016(09)

[8] Xu Wenhui, Tang Lizhou. Research on the "Four Desirable" Strategy in Beautiful Countryside Planning and Construction [J]. Chinese Landscape Agriculture. 2016(09)

[9] Xu Shan, Huang Biao, Liu Xiaoming, Zhang Yujun. From Perception to Cognition Characteristics of Rural Landscape Style in Beijing [J]. Landscape Architecture. 2013(08)

[10] Wang Nanxi, Lu Qi. Research on Rural Landscape Value Evaluation Factors and Sustainable Development Method [J]. Landscape Architecture. 2015(12)

[11] Zhang Xiaotong, Liu Wenping, Xiao He. Zhang Qian, Yu Zhenrong. Scenario Visualization Assessment of Rural Landscape base on Visual Indicators with Ecological Significance [J]. Chinese Journal of Eco-Agriculture 2017(08)

[12] Yang Zhijie. Research and Evaluation of Rural Settlements Landscape in Shanghai [D]. 2009:63-69 